\title{
Two-fluid model to simulate metal powder bed fusion additive manufacturing
}

\author{
Noriko WATARI*, Yuzuru OGURA*, Noriko YAMAZAKI*, Yukihiko INOUE*, \\ Keisuke KAMITANI*, Yasuyuki FUJIYA*, Masahiko TOYODA*, Saneyuki GOYA* and \\ Toshiya WATANABE* \\ ${ }^{*}$ Research \& Innovation Center, Mitsubishi Heavy Industries, Ltd. \\ 3-3-1, Minatomirai, Nishi-ku, Yokohama, 220-8401, Japan \\ E-mail: noriko_watari@mhi.co.jp
}

Received: 27 December 2017; Revised: 5 April 2018; Accepted: 24 August 2018

\begin{abstract}
This paper reports our simulations of the metal powder fusion additive manufacturing process based on a two-fluid model. In simulations of metal behavior in which heat is applied by high-density energy sources (e.g., laser or electron beam), the aspects that need to be correctly modeled include boiling and evaporation, as well as melting and solidification. The potential of the two-fluid model to clarify numerous physical phenomena-deep penetration, plume generation, spatter generation due to interfacial pressure differences between gas and liquid phases, and the transport of spatter with high-speed-plume flow-are shown from a perspective that departs from conventional interpretations. This paper compares and discusses the results of simulating the penetration and width of single beads and experimental results.
\end{abstract}

Keywords : Additive manufacturing, Two-fluid model, Evaporation, Plume, Spatter, Heat counterflow, Knudsen layer

\section{Introduction}

Metal additive manufacturing (AM) is a metal molding process that involves the repeated laying of powdery metal and the fusion-solidification in a selected area. Since the melting region and layer-by-layer deposition are controlled using digital computer-aided design data, AM is also called three-dimentional printing. A laser or electron beam is used to melt the metal. AM is among the most intensely competitive fields in manufacturing process developments. Therefore, despite very complicated physical phenomena, considerable research based on simulation has been done. We would like to give reviews to, for example, (King et al.,2015, Khairalla et al., 2016, and Ly et al., 2017). The targets of these studies can be roughly divided into three areas: (1) solving thermal balance, (2) solving thermal strain, and (3) expectation of crystal phase and structure (Sahoo and Chou, 2016). Approaches to the first item is further subdivided into (i) microscopic and (ii) mesoscopic methods. In the microscopic method, fluid-based simulations are performed on a metal powder scale of resolution, or several tens of $\mu \mathrm{m}$. In the mesoscopic method, the pile of powder is treated as a homogeneous continuum body with effective thermo-mechanical properties. For the type of (1)-(ii), heat transfer simulation code based on the finite element method (FEM) is often used, and the liquid properties of molten metal are ignored. This study aims for first-principles calculation of type (1)-(i), without using specially elaborated models.

Figure 1 gives an overview of physical model for AM by laser. Such a physical model is also adopted by other research groups(Klassen et al. 2014). It is quite common to use a fluid model for metal fusion-solidification simulation. The crucial difference in this study from other researches is the use of two-fluid model. The two-phase flow and two-fluid model are sometimes confused. Two-phase flow is a model dealing with gas and liquid, but with no specification concerning the number of vectors of field. The two-fluid model has two vector fields of flows. This method features vectors of two streams that differ in direction within a spatially identical volume. For this study we use 
Advance/FrontFlow/MP, a commercially available two-fluid software based on finite volume method (FVM). This software was originally developed to simulate nuclear power system on meter scale, but in this research remodeled to deal with micro-fluid and metal fusion-solidification-evaporation.

Figure 2 shows the forces acting on the fluid, which vary depending on the representative length. For the estimation of the force, the physical property value of molten iron and the velocity of fluid were calculated using 100 $\mathrm{m} / \mathrm{s}$. While the velocity of fluid is very large, the relative speed of the gas phase when metal vapor is generated is this order. The viscous force is small and the Reynolds number is about $10^{4}$. In micro-fluids, the effect of surface tension has an overwhelming influence. The Marangoni force derived from the temperature dependence of surface tension is also important. The surface tension is such that the accuracy is insufficient for gas-liquid interface capture by volume of fluid (VOF) method.

To perform a simulation at powder scale resolution, the spherical shape of the metal alloy powder must be captured by the box cell. The powder diameter is $20 \mu \mathrm{m}$ for small ones and about $100 \mu \mathrm{m}$ for large ones. The special mesh size is required to be $2 \mu \mathrm{m}$ to $4 \mu \mathrm{m}$. Since the length of the molten pool is about $1 \mathrm{~mm}$, the number of meshes in the scanning direction must be at least 250 and the total number of box cells must be at least 20 million necessary. Although it cannot be said that it is big as a fluid dynamics model today, the fineness of the space mesh size requires a fine time step size - typically, about from $10^{-8}$ to $10^{-9}$ seconds. With time increments on this scale, the number of time increments required to scan the size of the molten pool length at a typical scanning speed of $400 \mathrm{~mm} / \mathrm{s}$ is around 250,000 . The resulting model places massive computing demands for the axis of time and can run only on a super computer. For such calculations, the effect of the interconnection performance is greater than the CPU performance.

In the research discussed in this paper, we simulated the phenomenon of melt-solidification of a stack of metal powders on the micrometer scale by local moving heat sources such as laser beam for the metal additive manufacture of the powder bed type on a millimeter scale model. Our goal to understand the basic physical phenomena underlying melting and solidification based on behavior observed at micrometer resolution. In addition, we sought to quantitatively predict appropriate process conditions depending on the properties of materials and powders. We present the physical model and method in section 2, the simulation model and results in section 3 and experiments for validation in section 4. Section 5 provides further discussion..

\section{Physical model and method}

The physical model presented here for simulating laser additive manufacturing is implemented in the framework of an FVM-based commercial software for the two-fluid model, Advance/FrontFlow/MP. The basic two-fluid model consists of a metal phase and a gas phase. Metal solid state is represented by changing the viscosity according to the temperature as a special form of liquid. The melting and solidification have been specially implemented with the latent heat model. The evaporation is treated by the mass generation model provided in the native two fluid model .

\subsection{Two-fluid model}

Gas-liquid two-phase flow is treated with the Eulerian-Eulerian two-fluid model. The governing equations related to the velocity and pressure of the incompressible fluid are solved by the semi-implicit method for pressure-linked equation (SIMPLE) method. Time integrals are evaluated by the implicit Euler method.

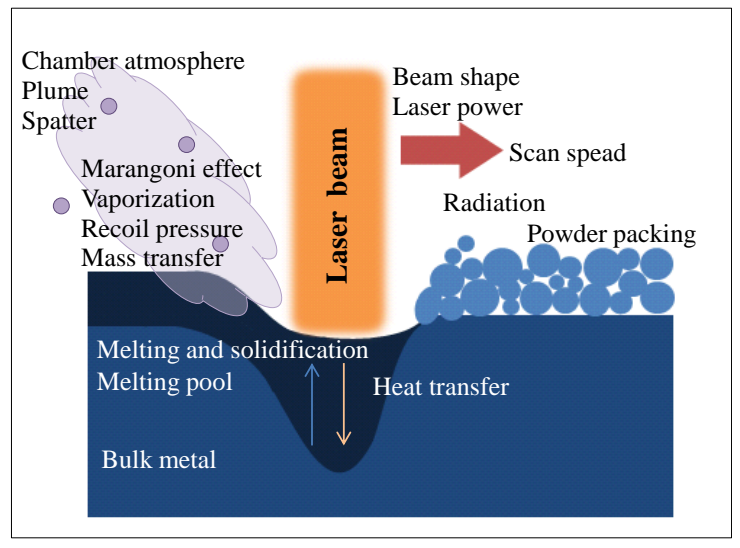

Fig. 1 Overview of model for simulating laser additive manufacturing process.

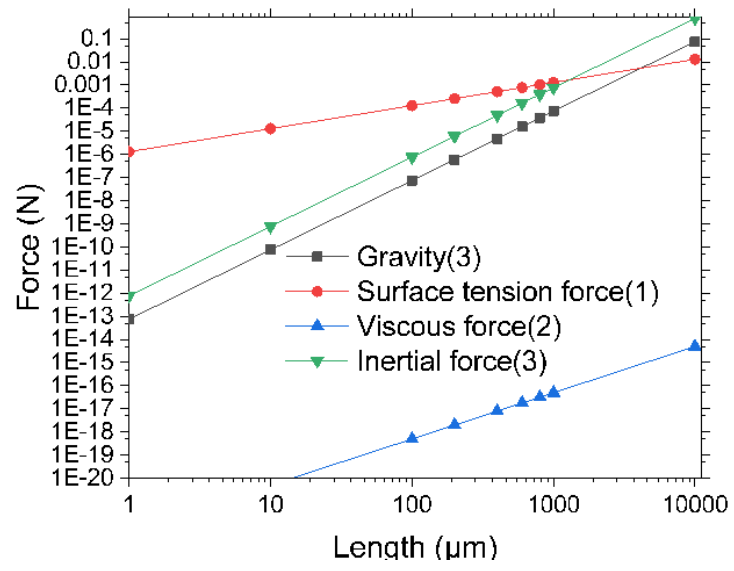

Fig. 2 Forces acting on the fluid. Dimensions of forces are shown in parentheses. The physical property values of molten iron are used for estimation. 
Watari, Ogura, Yamazaki, Inoue, Kamitani, Fujiya, Toyoda, Goya and Watanabe,

Journal of Fluid Science and Technology, Vol.13, No.2 (2018)

Table 1 Notations used in equations (1)-(7).

\begin{tabular}{c|l|c}
\hline \hline Notation & \multicolumn{1}{|c}{ Meaning } & Dimension \\
\hline$k$ & indicates phase; k=l liquid, k=g gas & - \\
$\rho_{k}$ & Density of phase k & $\mathrm{kg} / \mathrm{m}^{3}$ \\
$\alpha_{k}$ & Volume fraction of phase k & - \\
$v_{k}$ & Velocity of phase k & $\mathrm{m} / \mathrm{s}$ \\
$\Gamma_{k}$ & Mass production ratio of phase k & $\mathrm{kg} / \mathrm{s} / \mathrm{m}^{3}$ \\
$\tau_{k}$ & Shear stress of phase k & $\mathrm{N} / \mathrm{m}^{2}$ \\
$F^{\prime}{ }_{w k}$ & Wall friction force of phase k & $\mathrm{N} / \mathrm{m}^{3}$ \\
$F_{i k}$ & Interface friction force & $\mathrm{N} / \mathrm{m}^{3}$ \\
$F_{b k}$ & Interface friction force in babbleflow & $\mathrm{N} / \mathrm{m}^{3}$ \\
$F_{g k}$ & Volumetric force including gravity of phase $\mathrm{k}$ & $\mathrm{N} / \mathrm{m}^{3}$ \\
$g$ & Gravitational acceleration & $\mathrm{m} / \mathrm{s}^{2}$ \\
$P$ & Static pressure & $\mathrm{N} / \mathrm{m}^{2}$ \\
$h_{i k}$ & Saturation enthalph of phase k & $\mathrm{J} / \mathrm{kg}$ \\
$h_{k}$ & Enthalpy of phase k & $\mathrm{J} / \mathrm{kg}$ \\
$q_{k}$ & Heat flux of phase k & $\mathrm{W} / \mathrm{m}^{2}$ \\
$q_{w k}$ & Heat flux of phase k at wall & $\mathrm{W} / \mathrm{m}^{2}$ \\
$C_{D}$ & Drag coefficients of single bubble & - \\
$d$ & Averrage bubble diameter & $\mathrm{m}$ \\
$v_{r}$ & Relative velocity between gas and liquid & $\mathrm{m} / \mathrm{s}$ \\
$E_{O}$ & Eötvös number & - \\
\hline & & \\
\hline
\end{tabular}

The governing equations are given below. A vector is represented by superscript arrow, a tensor by a supperscript double line, and a matrix by an underline.

(1) Mass conservation equations for gas phase and liquid phase:

$\frac{\partial}{\partial \mathrm{t}}\left(\rho_{k} \alpha_{k}\right)+\nabla \cdot\left(\rho_{k} \alpha_{k} \vec{v}_{k}\right)=\Gamma_{k}$,

$\Gamma_{l}=\Gamma_{g}$,

(2) Momentum conservation equations for gas phase and liquid phase:

$\frac{\partial}{\partial \mathrm{t}}\left(\rho_{k} \alpha_{k} \vec{v}_{k}\right)+\nabla \cdot\left(\rho_{k} \alpha_{k} \vec{v}_{k} \vec{v}_{k}\right)=-\nabla \cdot\left(\alpha_{k} \overline{\bar{\tau}}_{k}\right)-\vec{F}_{i k}-\vec{F}_{g k}-\alpha_{k} \nabla \mathrm{P}+\Gamma_{k} \vec{v}_{i k}-\vec{F}_{v}^{s t}-\vec{F}_{v}^{m a}$

(3) Energy conservation equations for gas phase and liquid phase:

$\frac{\partial}{\partial \mathrm{t}}\left(\rho_{k} \alpha_{k} h_{k}\right)+\nabla \cdot\left(\rho_{k} \alpha_{k} \vec{v}_{k} h_{k}\right)=-\nabla \cdot\left(\alpha_{k} \vec{q}_{k}\right)+\alpha_{k} \frac{\mathrm{DP}}{\mathrm{Dt}}+\Gamma_{k} h_{i k}$.

The notations used in these equations are tabulated in Table 1. Although the momentum across the gas-liquid interface is preserved, due to the constraints of an incompressible fluid, it is not possible to calculate the recoil pressure resulting from the expansion of the gas accompanying the phase change.

In the two-fluid model, as with the VOF method, each liquid and gas present in one calculation cell is represented by a volume fraction. The flow mode is defined from the gas-liquid fraction. Depending on the flow mode, model expressions for such as interface friction and interfacial heat transfer are used. In this study, calculations are performed using the flow mode model of liquid single phase - bubble flow - gas single phase. Based on examination after performing the calculations, we found that the flow mode that transits to bubble flow - transition flow - spray flow is appropriate. In the bubble flow mode, the interfacial friction force of the following formula is applied (Tomiyama et al., 1998). This is a drag coefficient formula used when $E_{o}$ is low and the surface tension is dominant. The Eötvös number of liquid iron is about $5 \times 10^{-4}$ when the representative length is $100 \mu \mathrm{m}$.

$\vec{F}_{i k}=\frac{3}{4} \frac{C_{D} \alpha_{g}}{d} \rho_{l}\left|\vec{v}_{r}\right| \vec{v}_{r}$ 
$C_{D}=\frac{2}{3} E_{o}$

As can be seen from Fig. 2, since the surface tension dominates over the viscosity, we use the drag coefficient of the Eötvös number definition. In this simulation, the heat transfer between phases is not considered. The average bubble diameter is fixed at $30 \mu \mathrm{m}$. The flow mode model used here presents the problem that the interfacial frictional force becomes discontinuous at the transition between the bubble flow and the gas single phase. We think that the flow mode model of bubble flow - transition flow - spray flow can eliminate this discontinuity.

For a fluid with a representative length of the system on the order of micrometers, the surface tension is about 100,000 times greater than the gravity. We used the following procedure to calculate the surface tension as a volumetric force.

$\vec{F}_{v}^{s t}=\sigma \kappa \nabla \alpha_{l}$,

where $\sigma$ is surface tension, and $\kappa$ is the curvature of the gas-liquid interface as defined by the following equation.

$\kappa=-\nabla \cdot \vec{n}$

Hrere, $n$ is an interface normal vector from the gas phase to the liquid phase. $F_{V}^{S T}$ is added to $F_{g k}$. Accuracy in the capture of the curvature of the gas-liquid surface is critical in calculating the surface tension. In the two-fluid model, the volume fraction of liquid is advected by time evolution. An isosurface with liquid volume fraction of 0.5 corresponds to the gas-liquid interface. Solving the Hamilton-Jacobi equation makes it possible to capture the isosurface smoothly.

The Marangoni force, a difference in surface tension force attribute to temperature difference and a characteristic force of a micro-fluid, is incorporated as a volumetric force by the following equation.

$\nabla_{s}=\left(\underline{I}-\vec{n} \vec{n}^{T}\right) \nabla$

$\vec{F}_{f}^{m a}=\nabla_{s} \sigma=\left(\underline{I}-\vec{n} \vec{n}^{T}\right) \nabla \sigma=\frac{\partial \sigma}{\partial T}\left(\underline{I}-\vec{n} \vec{n}^{T}\right) \nabla T$,

$\vec{F}_{v}^{m a}=\vec{F}_{f}^{m a} \delta\left(\vec{x}-\vec{x}_{s}\right)=\frac{\partial \sigma}{\partial T}\left(\underline{I}-\vec{n} \vec{n}^{T}\right) \nabla T \delta\left(\vec{x}-\vec{x}_{s}\right)$.

Here $\nabla_{\mathrm{S}}$ is surface projection tensor, $\underline{I}$ the identity matrix, $\vec{n}$ the unit normal vector to the gas-liquid interface, $\sigma$ the surface tension force, $T$ temperature, $\vec{x}$ spatial coordinate, and $\vec{x}_{S}$ is coordinate of the interface. The absolute value of Marangoni force is about $20 \%$ of absolute value of surface tension force.

\subsection{Phase changes}

Two phase changes occur in the AM metal molding process; one is a solid-liquid change and another is a liquid-vapor change. The solid-liquid change is implemented by the enthalpy method. The enthalpy h of solid phase, liquid phase, solid-liquid mixed phase is defined as follows.

$h=C_{P, S} T \quad($ solid $)$,

$h=C_{P, L}\left(T-T_{S}\right)+h_{L, S} \quad$ (liquid),

$h=\left\{C_{P, S}(1-f)+C_{P, L} f\right\} T+\left(h_{L, S}-C_{P, L} T\right) f \cdot($ solid-liquid mixed)

$C_{P, S}$ is the constant pressure specific heat of the solid, $C_{P, L}$ is the constant pressure specific heat of the liquid, $T_{S}$ is the melting point, and $h_{L, S}$ is the saturation enthalpy of the liquid. The enthalpy $h$ is determined from the heat balance, temperature $T$ and liquid phase fraction ratio $f$ are defined based on enthalpy.

$$
f=\frac{h-h_{S, S}}{h_{L, S}-h_{S, S}} .
$$

$f=0$ is the solid phase. $\mathrm{h}_{\mathrm{s}, \mathrm{s}}$ is the saturation enthalpy of the solid. $h_{L, S}-h_{S, S}$ corresponds to the latent heat of melting. The flow of the solid phase is fixed by setting the viscosity $\mu_{\mathrm{s}}$ to a large number. $\mu_{\mathrm{L}}$ is viscosity of liquid. In this study $\mu_{\mathrm{s}}$ is set to $1 \times 10^{5}$. The viscosity in the solid-liquid mixed state is defined below,

$$
\mu=f \mu_{L}+(1-f) \mu_{S} \text {. }
$$

The two-fluid model inherently accounts for the liquid-vapor phase change. In this study only the phase change from liquid to vapor is considered. For metal alloys, the mass production rate for gas phase $\Gamma \mathrm{g}$ in equations (1), (3) and 
(4) is given by Ansimov's formula,

$$
j=P_{a} \cdot \frac{4.32}{3 \pi} \sqrt{\frac{m}{2 \pi k_{B} T}} \exp \left(-\frac{\lambda}{k_{B}}\left(\frac{1}{T}-\frac{1}{T_{B}}\right)\right)
$$

$\Gamma_{g}=j \Delta_{s}$

Here $j$ is mass flux of liquid phase to gas phase, $P_{a}$ is the standard atmospheric pressure, $\mathrm{m}$ is atomic weight, $k_{B}$ is Boltzmann constant, $\mathrm{T}$ is absolute temperature, $\lambda$ is cohesive energy, $T_{B}$ is the boiling point at standard atmospheric pressure, and $\Delta S$ is the area of a side surface of the cubic calculation cell. The amount of evaporation calculated by Ansimov's formula shows good agreement with values mesured with the high temperature metal thermophysical property measurement system by the electromagnetic levitation method (PROSPECT) (Fukuyama, 2015, and Watanabe et al., 2016) in the temperature range up to 2,000 K. In PROSPECT, the metal sample is melted in a levitated state and its volume is determined from a photograph of the sample cross section. The temperature-depent density is calculated using the average mass of the sample before and after levitation and the above mentioned volume. After determining the density, the mass change when holding for a certain period of time at a predetermined temperature is measured from the shadow image of the sample. At the same time, the rate of evaporation per unit of surface area is obtained based on the assumption of a perfect sphere. The particulars of this method will be presented in another paper by Fukuyama et al.

One features of the two-fluid model is that mass production is taken into account for all liquid volumes. The native mass generation model for the two fluid model preserves conservation law of mass, momentum and energy traversing gas-liquid interface, but recoil pressure cannot be estimated. The origin of the recoil force is the pressure accompanying the expansion of the gas and cannot be described by the equation for the incompressible fluid which does not incorporate gas density change as a model. This point is not a first principle. The following recoil pressure is introduced artificially (Khairallah et al., 2016).

$$
P(T)=0.54 P_{a} \exp \left(-\frac{\lambda}{k_{B}}\left(\frac{1}{T}-\frac{1}{T_{B}}\right)\right),
$$

Multiplying the $\delta$ function of the interface and the element cell volume to recoil pressure, we convert it into the volume force $F_{\text {recoil }}$, whose direction is normal to the liquid interface. $F_{\text {recoil }}$ is added to $F_{g l}$ in equation (3).

\subsection{Laser irradiation and thermal radiation}

Heat input $I(x, y)$ is applied to the surface of the metal, with Gaussian intensity distribution in the plane perpendicular to irradiation direction $\mathrm{z}$.

$$
\begin{aligned}
& I(x, y)=\frac{2 W}{R^{2} \pi} \exp \left(-\frac{2 r^{2}}{R^{2}}\right), \\
& r=\sqrt{x^{2}+y^{2}} .
\end{aligned}
$$

Here $W$ is laser power multiplied by the absorption coefficient, $R$ is the $e^{2}$ radius of the beam, and $(x, y)$ are in-plane coordinates with the beam center as the origin. Laser energy is expressed in terms of intensity per area; in the finite volume method this is converted to energy per volume. When the volume fraction of the metal in the surface box cell is small, the heat input density per volume becomes large; in contrast, when the volume fraction is large, the heat input density becomes small, generating an unnatiral distribution like the annuarl rings in Fig.3(a). To avoid this, we searched in the vertical direction and take the thickness until the sum of the volumetric proportions of the metal in the cell volume becomes 1 and distribute the heat according to the surface area occupied by the plane perpendicular to the heat input direction, as shown in Fig. 3(b).

The effective heat input is further multiplied by laser absorption rate. The heat of $\sigma \varepsilon T^{4}$ is lost on the surface of metal by radiation. Here, $\sigma$ is the Stefan-Boltzmann coefficient and $\varepsilon$ is emissivity. Laser absorption is equal to emissivity..

\subsection{Two-fluid model procedure}

The time development procedure of the whole two-fluid calculation is carried out as follows:

(1)At each time step and self-consistent cycle, four regions of solid, liquid single phase, gas-liquid mixed phase, and gas single phase are determined for each calculation mesh.

(2)In the gas-liquid mixed phase, the mass, momentum, and energy transported across the gas-liquid interface are calculated.

(3)For each phase of gas and liquid, we solve the equation of motion for the fluid.

(4)By solving the Poisson equation for pressure, we correct the pressure (the same value for the gas and the liquid) and 
the velocities for the gas phase and the liquid phase.

(5)Solve mass conservation equations for gas phase and liquid phase.

(6)Solve energy conservation equations for gas and liquid phases.

(7)Solve heat conduction equation for the solid.

The procedure specified in (1) - (7), solves for the variables of the pressure, the velocity of each phase, the enthalpy of each pase, and the volumetiric ratio of each phase. The cycle of (1)-(7) is repeated until these variables become self-consistent. After convergence, the calculation for the next time step starts.

(a)
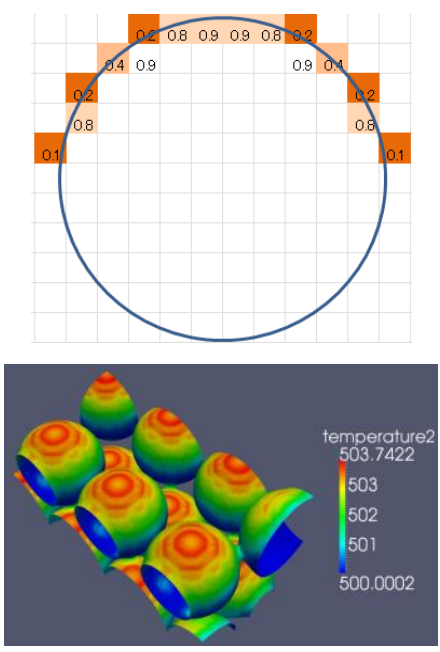

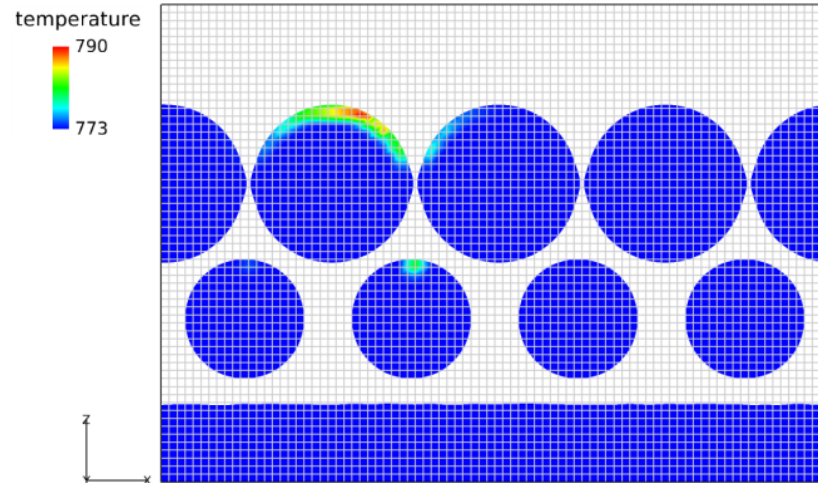

(b)

Fig. 3 (a) Difference in energy density per volume caused by approximating a sphere with cubic cells, and energy density distribution like annual rings. The numbers in the upper panel are the volume fraction of metal in the cell. (b) Sectional view of modified laser heat input. Color indicates temperature.

Table 2 The physical property values of Inconel718 used in this simulation. * Experimental value, ** calculated arithmetically in terms of composition element ratios.

\begin{tabular}{ll|c|c}
\hline \hline & Physical property & Value & Dimension \\
\hline Fluid & Density & 7619.635 & $\mathrm{~kg} / \mathrm{m} 3$ \\
& Viscosity & $8.9958 \times 10^{-3}$ & $\mathrm{~Pa} \mathrm{~s}$ \\
\multirow{5}{*}{ Thermophysics } & $-0.0003 \times \mathrm{T}+2.1943$ & $\mathrm{~N} / \mathrm{m}$ \\
& Surface tension & $510.13 \times \mathrm{T}-178801$ & $\mathrm{~J} / \mathrm{kg} / \mathrm{K}$ \\
& Specific heat of solid & 727.72 & $\mathrm{~J} / \mathrm{kg} / \mathrm{K}$ \\
& Specific heat of liquid & $0.0145 \times \mathrm{T}+7.5194$ & $\mathrm{~W} / \mathrm{m} / \mathrm{K}$ \\
\multirow{4}{*}{ Melting } & Thermal conductiivity & $0.3 *$ & - \\
& Laser absorption ratio & 1493.16 & $\mathrm{~kg} / \mathrm{m} 3$ \\
Evaporation & Melting point & $2.5748 \times 10^{5}$ & $\mathrm{~J} / \mathrm{kg}$ \\
& Latent heat of melting & $3303 * *$ & $\mathrm{~K}$ \\
& Boiling point & $6.218 \times 10^{6 * * *}$ & $\mathrm{~J} / \mathrm{kg}$ \\
\hline
\end{tabular}

\section{$0.87 \mathrm{~mm}$}

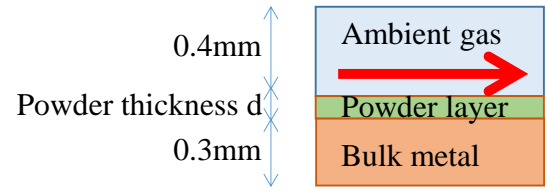

(a)

Fig. 4 The FVM model used in this study: (a) side view, (b) top view. $0.67 \mathrm{~mm}$ is the length of beam scan.
Table 3 Diameter distribution of inconel718 powder model.

\begin{tabular}{c|c}
\hline \hline $\begin{array}{c}\text { Diameter } \\
\mu \mathrm{m}\end{array}$ & $\begin{array}{c}\text { Frequency } \\
\text { probability }\end{array}$ \\
\hline 20 & 0.55 \\
30 & 0.393 \\
40 & 0.057 \\
\hline
\end{tabular}




\section{Simulation model and results}

\subsection{Physical property values}

The thermal properties and fluidity properties of the material alloy must be modeled to simulate melting, solidification, and evaporation phenomena by AM. In this study, the calculations assume a model based on Inconel718 alloy. Table 2 gives the values assumed. Values other than boiling point, latent heat of evaporation, and laser absorption rate are obtained from a database software of physical properties of metal alloys, JMatPro. (Sanders,2000, and Kijima, 2015)

With respect to surface tension and the specific heat of solid and the thermal conductivity, we assumed linear dependence on temperature. The boiling point and latent heat of vaporization cannot be obtained with JMatPro. These values were calculated by averaging of the known values for each element with weight by the molar ratio: $\mathrm{Cr}-19$, Ni-54, Mo-3, Fe-17, Co-1, Nb-2, Ta-3, Ti-1. The ambient gas is Ar. Density is $1.784 \mathrm{~kg} / \mathrm{m}^{3}$, the viscosity coefficient is $2.2 \times 10^{-5} \mathrm{~Pa} \mathrm{~s}$, the specific heat is $520.32 \mathrm{~J} / \mathrm{kg} / \mathrm{K}$, and the thermal conductivity coefficient is $1.772 \times 10^{-2} \mathrm{~W} / \mathrm{m} / \mathrm{K}$.

\subsection{Powder deposition model}

The powder filling model was prepared using the distinct element method (DEM) integrated into Advance/REVOCAP/Porous_Modeler. In the DEM, powder and metal substrate are treated as geometric information. These are converted to a set of finite volume elements of hexahedral elements that can be used for fluid analysis, and are assigned as gas or metal attributes. Table 3 gives the particle size distribution in the powder bed, selected to model the experimental conditions as realistically as possible. The packing rate of the powder obtained by simulation is $53 \%$, which is slightly smaller than the actually measured value of $58 \%$. The packing rate $\chi=\mathrm{M} / \rho \mathrm{V}$, using the apparent volume $\mathrm{V}$, the mass $\mathrm{M}$ of powder, and the density $\rho$ of the metal. We adjusted the coefficient of friction between powders in the DEM, to adjusted the packing rate to the experimental value. The thickness $d$ of the powder stacking $\mathrm{d}=75.5 \mu \mathrm{m}$ is determined from the molding pitch to lower the stage; $\mathrm{z}=40 \mu \mathrm{m}$, and $\chi$ by the following equation:

$\cdot d=\frac{z}{\chi} \cdot$

Figure 4 shows the FVM model used in this study. The mesh is a $2.5 \mu \mathrm{m}$ cubic cell. The total number of meshes is about 24 million. For Cartesian coordinates, the irradiation direction of the laser is assumed to be the $-z$ axis and the scanning direction of the laser is assumed to be the $x$ axis. The boundary conditions of the finite element model are as follows,

upper panel: $\quad \nabla P=0, T=773.15 \mathrm{~K}$,

side panels: $\quad v_{z}=0, T=773.15 \mathrm{~K}$,

bottom panel: $v_{x}=0, v_{y}=0, T=773.15 \mathrm{~K}$.

\subsection{Simulation and results}

Single bead molding processes are simulated by setting the beam diameter to $100 \mu \mathrm{m}$, the laser power to $19 \mathrm{~W}$, $78 \mathrm{~W}$, $118 \mathrm{~W}$, and setting the scanning speed to $400 \mathrm{~mm} / \mathrm{s}, 1600 \mathrm{~mm} / \mathrm{s}, 2400 \mathrm{~mm} / \mathrm{s}$. At the time we began the simulation, we estimated value absorption rates based on a search of the literature Later we obtained our own measurement results and changed the laser absorption rates. This explains the fractional laser power values. We used time increments of $10^{-9}$ to $10^{-8}$ seconds. As the evaporation intensed, it becomes necessary to make the time steps finer.

Calculations were done in parallel of 1,392 cores. The elapsed time per step was 12 seconds. Ultimately, to obtain the results, we needed to calculate more than 35,000 steps. This took about one week for one case.

Figures 5 and 6 show the results. Deepening penetration as scanning speed slows is well-simulated, and the scattering of the spatter of melting metal is observed. In the one-fluid model, although the waves of the molten metal surface can be expressed, the scattering of the spatter as shown in Fig. 5 cannot be simulated. There was no simulation result for the AM that showed penetration depth. In the welding simulation, the depth of penetration was expressed using an artificial model such as multiple scattering of light, but this cannot be applied to AM. We will discuss the reasons why these phenomena could be simulated in secsion 5 . 


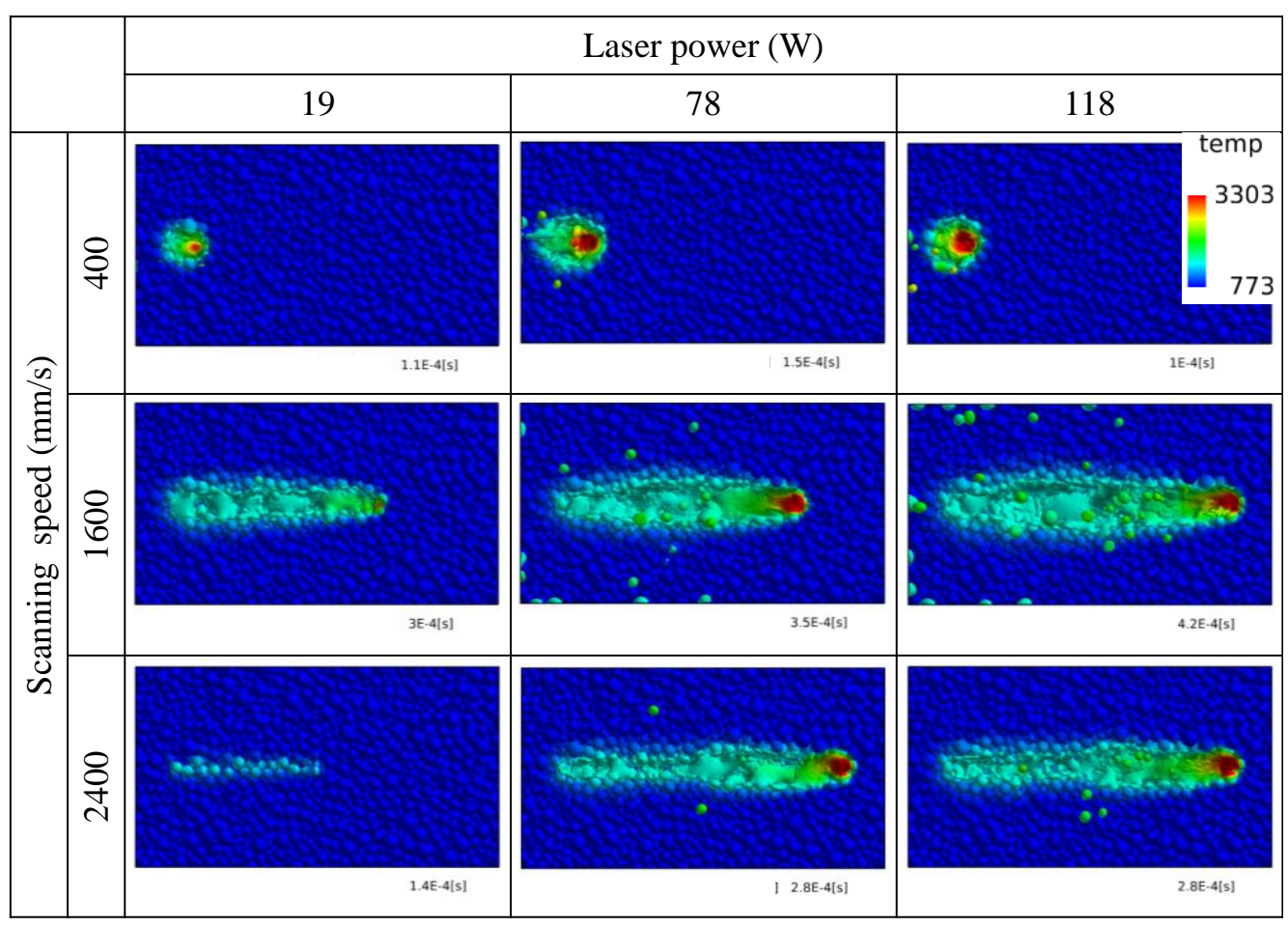

Fig. 5 Top view of single bead molding processes of Inconel718 by simulation. Temperature is indicated by color. It is possible to describe how the spatter scattering amount varies depending on the process conditions.

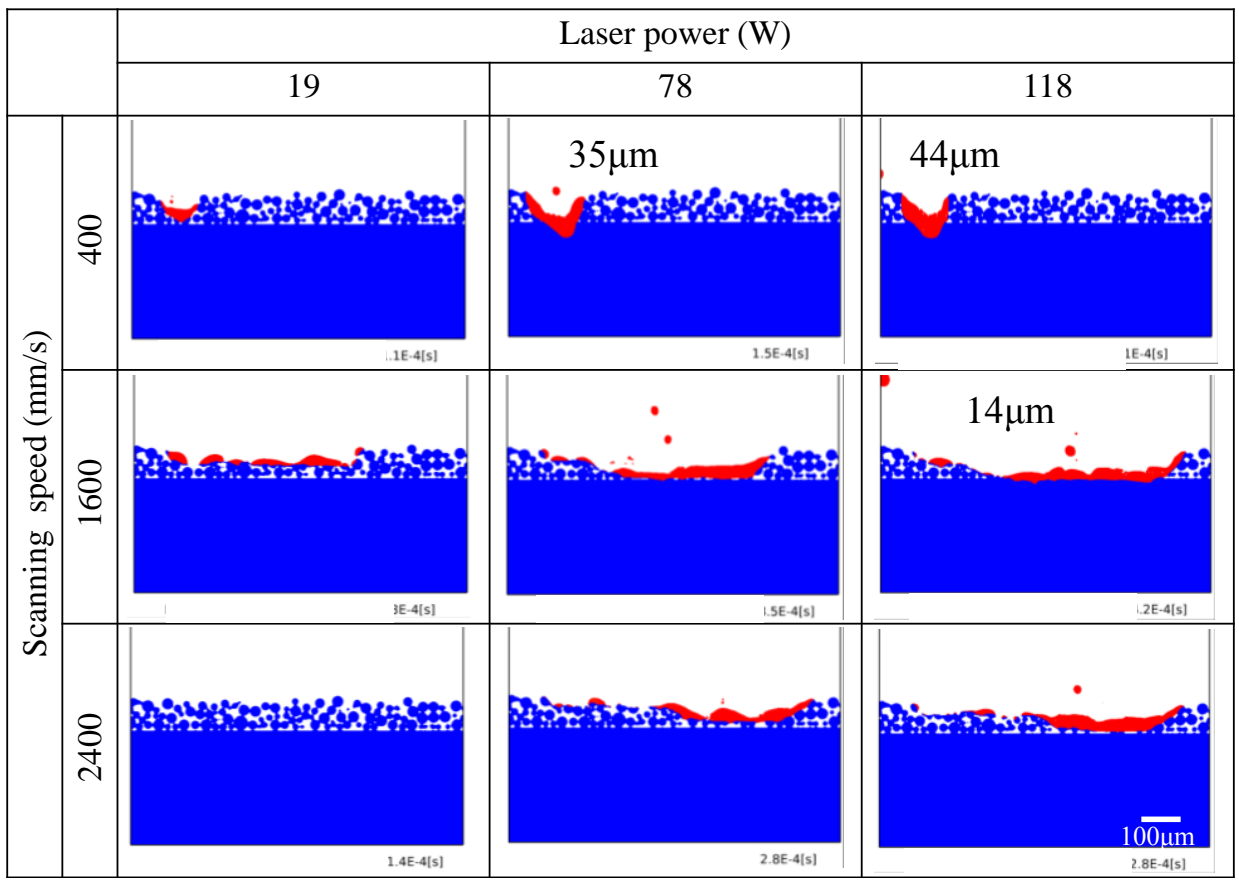

Fig. 6 Side view of single bead molding processes of Inconel718 by simulation. The melted part of the metal is indicated in red and the solid part in blue. The numbers in the figure indicate penetration depth.

\section{Experiments for verification}

\subsection{Observation of powder filling condition by synchrotron radiation}

An X-ray microtomography at the beamline 20XU (Experimental Hutch II) in the synchrotron radiation facility 
Super Photon ring 18 GeV (SPring-8) in Hyogo, Japan, was used for 3D structural observations.(Suzuki et al., 2000)

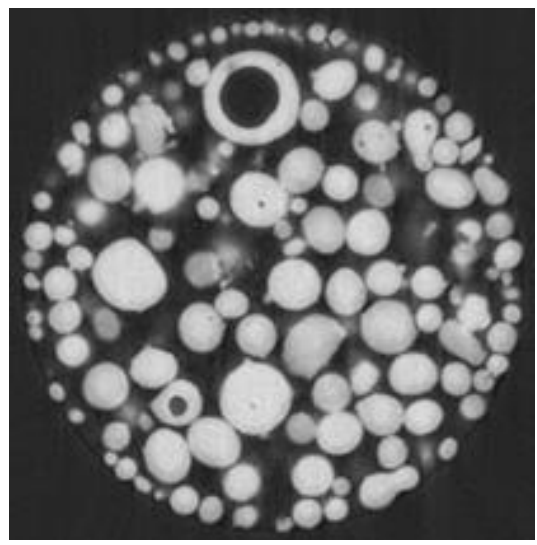

(a)

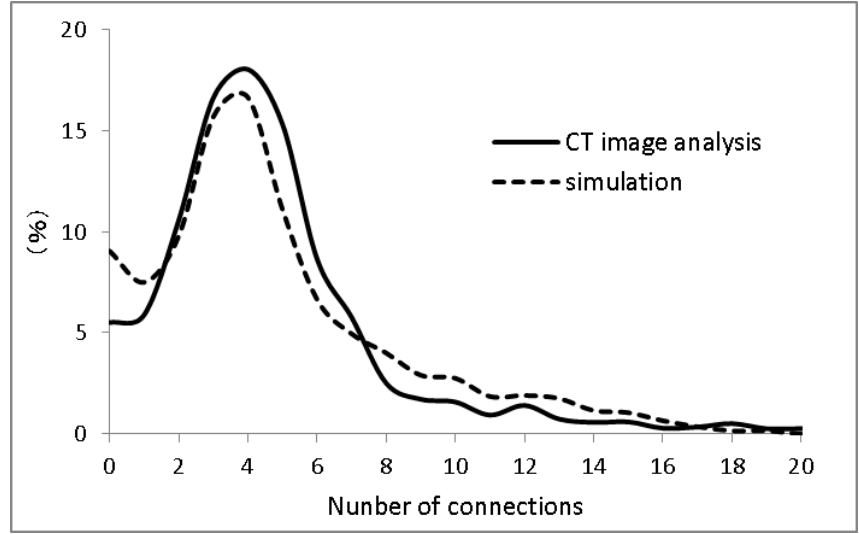

(b)

Fig. 7 (a) X-ray CT image with metal powder filled. (b) The analysis of the distribution of the number of connections between the metal particles from the X-ray CT image (solid line) and those analyzed from the filling by the simulation (dashed line) are compared.

The X-ray energy through a double-crystal monochromator was $37.7 \mathrm{keV}$. X-ray images at different angles were recorded using a complementary metal-oxide-semiconductor (CMOS) camera (Hamamatsu Photonics; ORCA Flash 4.0) with a visible-light conversion unit.The effective pixel size was $0.509 \mu \mathrm{m} / \mathrm{pixel}$. A total of 1,801 images were acquired with a rotation step of $0.20^{\circ}$ and an exposure time of $370.4 \mathrm{~ms}$. The recorded images were reconstructed to obtain tomograms, which were then stacked to generate 3D images as shown in Fig.7 (a).

Analysis of various data from 3D images was carried out mainly by using the AVIZO image analysis software (https://www.fei.com/software/amira-avizo) as follows: A threshold value is set for the gray scale of the CT image, binarization is performed, and surface extraction processing is performed to create a polygon mesh on the surface of the sphere. We separate sticking metal spheres in images by the Watershed method (Beucher, 1992) and label them. At this time, particle interlocking information shown in Fig. 7(b) is obtained by judging whether or not the adjacent labels are in contact with each other. Data such as the surface area, sphere equivalent diameter, barycentric coordinates, and so forth can be calculated from the volume of each label. Further, the filling rate can be obtained by calculating the ratio of the volume of the portion of the metal ball occupying the space.

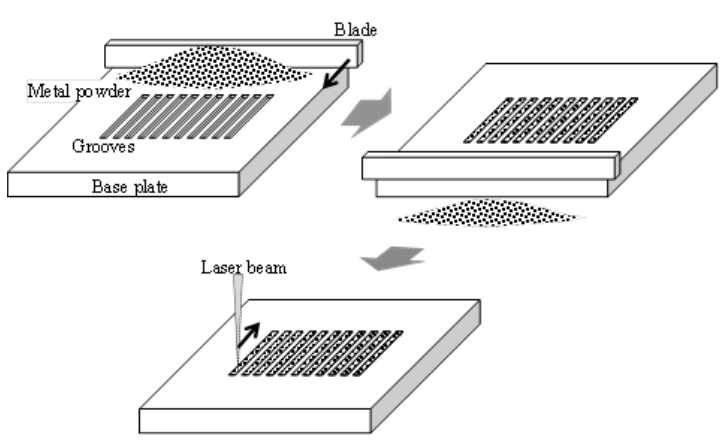

Fig. 8 Experimental procedure of forming single beads of metal powder.

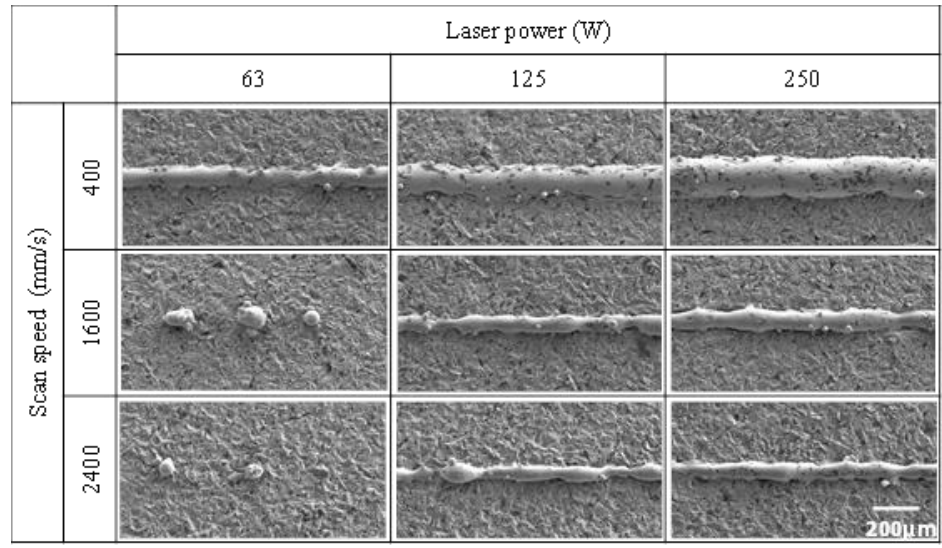

Fig. 9 Top views of single beads formed by laser irradiation with power range of $63 \mathrm{~W}$ to $250 \mathrm{~W}$ and scan speed range of $400 \mathrm{~mm} / \mathrm{s}$ to $2400 \mathrm{~mm} / \mathrm{s}$. 


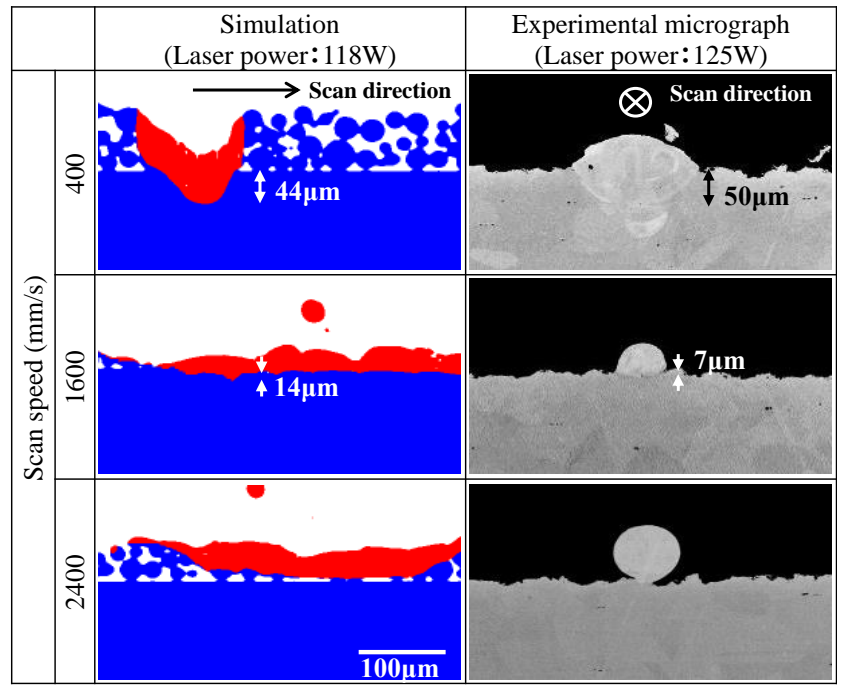

(a)

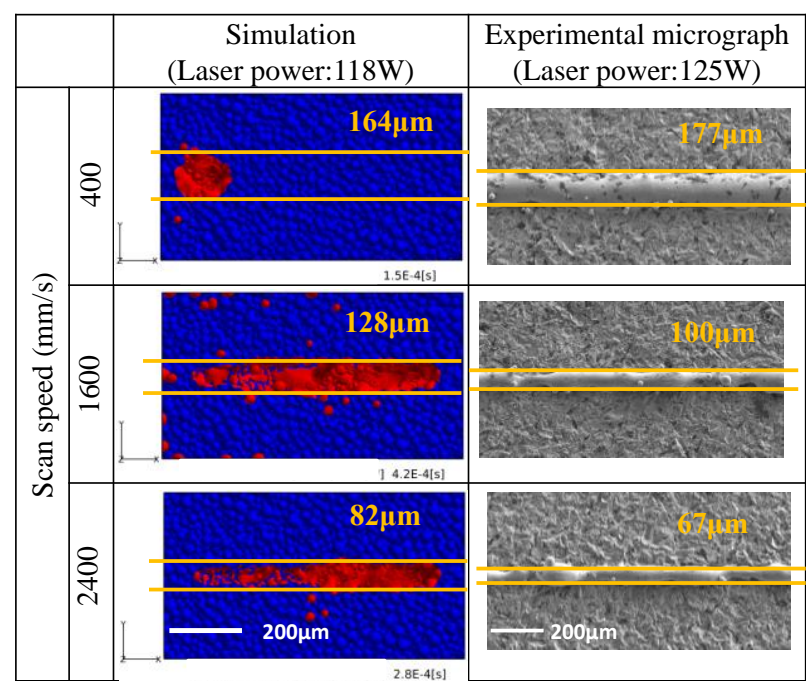

(b)

Fig. 10 (a) Comparison the melt depth predicted by the simulation and the experimental result for single bead formation. Left: side view of single bead molding processes of Inconel718 by simulation. The melted part of the metal is indicated in red and the solid part in blue. Right: the experimental results are shown with the scanning direction perpendicular to the page. (b) Top views for comparison between bead widths. Left: simulation results. The melted part of the metal is indicated in red and the solid part in blue. Right: experimental SEM image.

\subsection{Forming tests of single beads}

Single beads are formed on Inconel718 alloy powder filled in grooves with a depth of $0.1 \mathrm{~mm}$ on the base plate, by scanning a laser beam with a diameter of $0.1 \mathrm{~mm}$. Figure 8 illustrates the procedure for forming single beads. The Inconel718 alloy powder is EOS NickelAlloy IN718 with particle sizes under $70 \mu \mathrm{m}$. The laser beam was emitted by an EOSINT M290 with laser power of 63-370W. The scan speed was 400-2400 mm/s. Figure 9 provides SEM images of single beads viewed from directly above. Figure10 compares the depth of penetration into the substrate for the simulation and for the experiments.

In Figure 10(a), the output of the simulation shows a cross section parallel to scanning direction. A photograph of the experiment shows the section perpendicular to the scanning direction. The figure shows the laser scanning directions. The figures clearly show qualitative tendencies, that the penetration is formed at $400 \mathrm{~mm} / \mathrm{s}$, the melting metal wets at $1600 \mathrm{~mm} / \mathrm{s}$, and does not wet at $2400 \mathrm{~mm} / \mathrm{s}$, although the penetration depth error at $1600 \mathrm{~mm} / \mathrm{s}$ is $100 \%$. While this is large, since no calculation examples have compared penetration in the context of additive manufacturing simulations, this may be a good result. In Figure 10(b) compares the bead width for the simulation and the experiment. The value given by simulation is $20 \%$ to $30 \%$ greater than that by the experiment. These results are due to insufficient heat transfer in the depth direction due to the lack of a condensation model.

\section{Discussions}

Figure 11 shows temperature, the $\mathrm{z}$ component of the velocity vector of gas phase and the liquid phase in which sputter is projected. Figure 12 shows the pressure near the point of laser irradiation. Figures 11 and 12 show the results at a laser output of $118 \mathrm{~W}$ and scanning speed $400 \mathrm{~mm} / \mathrm{s}$ at $1.5 \times 10^{-4} \mathrm{~s}$. Figure 11 (c) shows how the evaporated metal forms a plume stream. The plume flow is derived as a natural result from the governing equation (1) of the two-fluid model. In this study, the maximum value of the fluid velocity was set to $100 \mathrm{~m} / \mathrm{s}$. The actual plume flow speed may be even faster.

We found a non-equilibrium region called the Knudsen layer at the gas-liquid interface where evaporation or condensation occurs (Klassen et al., 2014, Yano, 2008, and Takada, 2000), as shown in Fig. 12. Its spatial thickness is on the order of the mean free path $\lambda$ which can be calculated by the following formula,

$\lambda=k_{B} T /(\sqrt{2} P \sigma)$. 


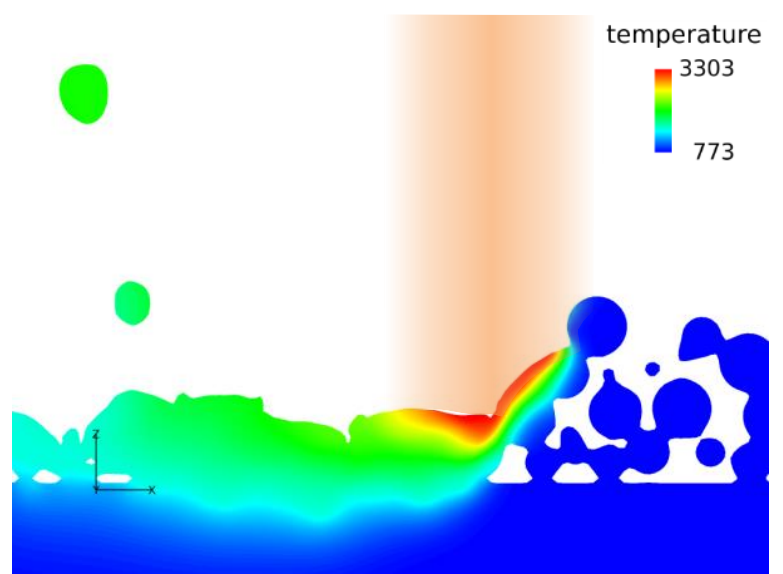

(a)

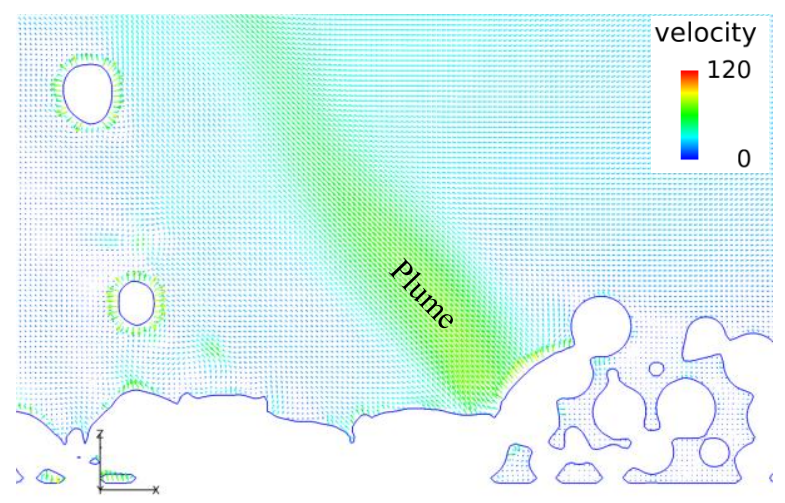

(c)

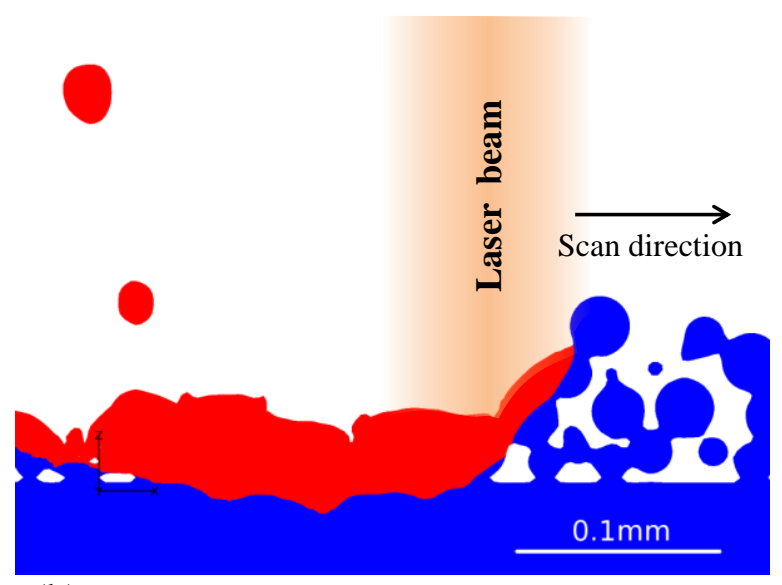

(b)

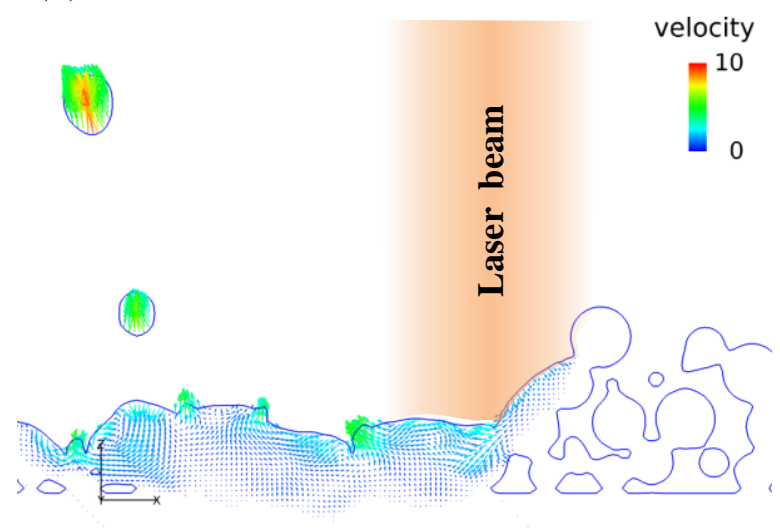

(d)

Fig. 11 Side view of melted state with sputtering: (a) temperature and (b) visualization of molten portion; the melted part is indicated in red and the solid part in blue, (c) and(d) z component of the velocity vector in melted state with sputtering, (c) for gas phase and (d) for liquid phase, respectively.

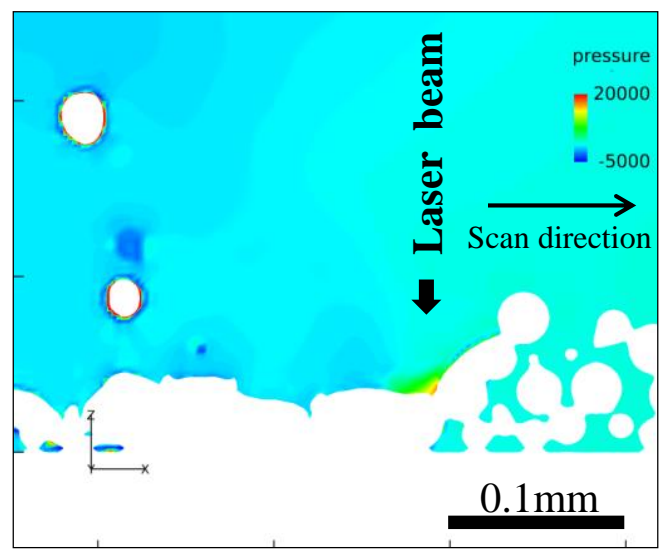

Fig. 12 Side view of pressure in gas phase with sputtering.

Here, $P$ is pressure and $\sigma$ is collision cross section; $\sigma$ can be approximated by $\sigma=\pi r^{2}$ using atomic radius r. By dividing the mean free path by thermal speed, $1,114 \mathrm{~m} / \mathrm{s}$, the mean free time $\tau$ can be determined. Around 3,000 K, the boiling point of metals at atmospheric pressure, the mean free path $\lambda$ of iron atom in the gas phase is about $6.3 \mu \mathrm{m}$ while mean free time $\tau$ is about $5.6 \times 10^{-9} \mathrm{~s}$. The Knudsen number $K_{n}$ of this system is 0.64 , which corresponds to an intermediate region between continuous fluid and molecular flow. The mesh size and time step used in the simulation are at the same scale as the spatial spread of the Knudsen layer and the mean free time of molecular flow. In figure 12, the thickness of the pressure fluctuation region of the vicinity of gas-liquid interface under evaporation is about 4 to $7 \mu \mathrm{m}$. The 
simulation model of this time can be considered to have captured the Knudsen layer with the minimum required resolution.

The speed of sound $\mathrm{c}$ in gas is given by

$$
c=\sqrt{\frac{\kappa R T}{M}},
$$

where $\kappa$ is specific heat ratio, $R$ is the gas constant, and $M$ is the average molecular weight. In iron vapor at 3,000 $\mathrm{K}$, this is $812 \mathrm{~m} \mathrm{~s}$. The thermal speed of iron gas is about 1.37 Mach. It exceeds the application limit of the equation of the incompressible fluid. The difference between the models of compressible fluid and incompressible fluid is the presence or absence of the gas state equation describing the relationship between density and pressure. In the two-fluid model, it is not possible to to describe the density change of the gas as a function of pressure or the change in pressure as a function of the density of the gas. The evaporative recoil force is a force that pushes the liquid interface in the process of expanding the vaporized gas.As a consequence, evaporative recoil force cannot be handled in the two fluid model. An artificial model is required.However, the two-fluid model describes the change in density when liquid changes into gas. Additionally, the pressure change at that time can be captured as a flowing momentum in a form across the calculation cell. This differs from the incompressible one-fluid model which is entirely incapable of modeling. It seems that it may be maintaining a global approximation of the Knudsen layer by interface treatment in the two-fluid model and appropriate special and time mesh sizes.

From these figures, we derive the following hypothesis concerning the mechanism of spatter flight is derived: (1) laser heat input melts concavo-convex surface, (2) there are temperature differences on the molten metal surface, (3) temperature differences generate differences in evaporation amount, (4) pressure difference arise due to the evaporation amount difference, (5) unevenness in liquid metal surface is induced by uneven pressure, (6) large surface tension force is applied and blunts the convex portion, and (7) the high-speed plume flow acts on the round protruding liquid portion, and the spatter is ejected in the direction opposite to direction of laser travel.

Since the laser enegy is light, heat input is only on the surface. Nevertheless, in a well-known phenomenon, heat is preferentially transferred in the depth direction. As an explanation of penetration depth for welding purposes, the multiple scattering of laser light and the generation of a keyhole has been cited as a cause (Tan et al. 2013). In contrast to welding, in the case of AM, the purpose is to fill the metal rather than to drill it, with a laser. The promotion of heat transfer by keyhole generation appears inexplicable.

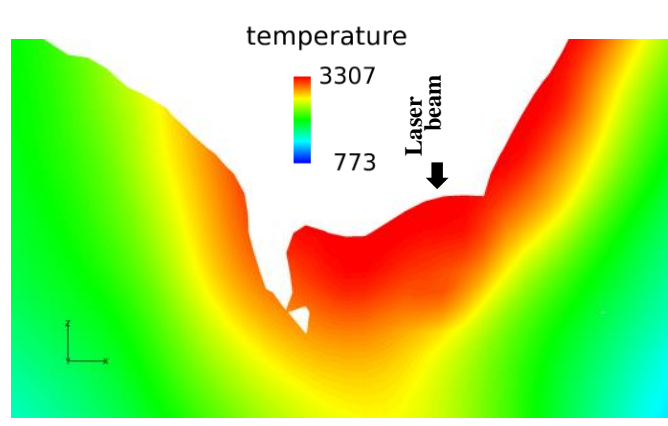

(a)

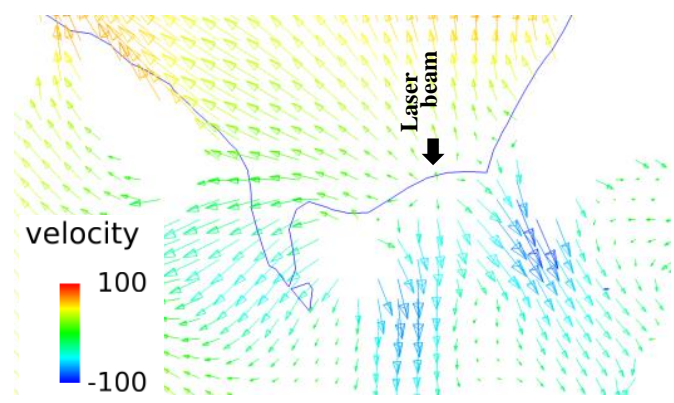

(c)

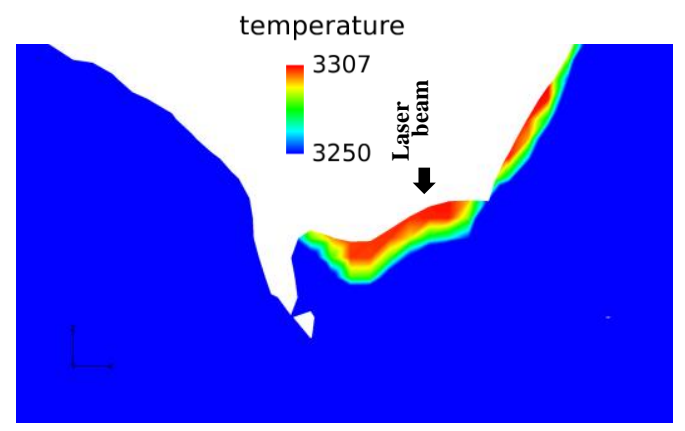

(b)

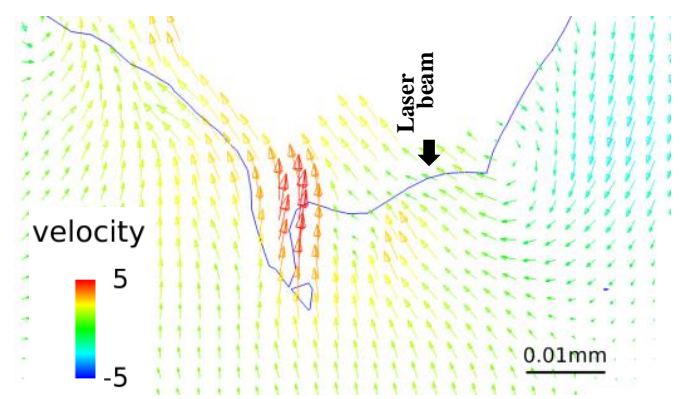

(d)

Fig. 13 Temperature and z-component of velocity distribution near laser irradiated part; (a) the whole temperature range of metal part, (b) high temperature range for laser irradiation area, (c) the z-component of velocity of gas phase, and (d) that of liquid phase. For velocities, cells with a volume ratio of 0.3 or more are displayed. 
Figure 13(a) and (b) show the temperature distribution near the laser irradiated portion. The laser is applied to the region with the highest temperature shown in Fig. 13 (b). Since all the metals in the region shown in Fig. 13 are at temperatures above the melting point, mass formation from liquid to gas occurs throughout the metal. That is, gas and liquid coexist across the whole area. The respective velocities of the gas phase and the liquid phase are shown in Fig. 13(c) and (d), respectively, for the same area of Fig. 13 (a) and (b). In the metal region just below the point irradiated by the laser, the liquid velocity is directing upwards, while gas velocity downwards. When the metal changes from liquid to gas, the flow of metal vapor also goes downward to satisfy the momentum conservation law. Liquid initially flowing with nearly zero velocity flows up as steam at very high speed, generating a large upward momentum. If there is no downward momentum, the conservation law is not satisfied. Furthermore, to satisfy the law of conservation of mass, the liquid metal lowing up must equal to the mass of the downward gas flow. The flow of steam with a large enthalpy goes down, while the flow of liquid with small enthalpy goes up. These two streams constitute a heat counterflow in which heat is efficiently transferred downward. This phenomenon is known as a heat transfer by heat counterflow in liquid helium (Guo et al., 2010). There is a difference between extremely low temperatures and ultrahigh temperatures in the temperature range. However, both helium and liquid metal are monoatomic molecules in the gaseous state, and heat counterflow occurs in their boiling states. For liquid only or gas only, heat counterflow becomes convection. Since the factor that drives convection is temperature difference, transmission speed is slow. Since this study does not incorporate a model of condensation, the speed of heat transfer is probably slower than what would be actually observed.

\section{Conclusion}

This paper discusses an exploration of fusion, coagulation and evaporation phenomena nvolving metal in an AM process based on a two-fluid model. This allows us to simulate the generation of plume flow and spatter which cannot be expressed by one-fluid model, and clarified the cause. We carried out molding experiments of single beads at varying scan speeds were performed. Tendency related to the depth of the molten pool observed in the experiments were in qualitative agreement with simulation results. Regarding the filling state of the powder, validity was verified by comparing simulation by the DEM and the observation by X-ray CT. One reason the heat applied by the laser is preferentially transmitted in the irradiation direction is believed to involve heat counterflow. We would like to plan improvements in predictive accuracy for heat balance by incorporating a model of condensation and consideration of the turbulence that accompanies heat counterflow.

\section{Acknowledgements}

The simulations presented here were carried out by adding microfluidic functions and a metal evaporation model to the Advance/FrontFlow/MP two-fluid commercial software and the powder stacking model to the Advance/REVOCAP/Porous-modeler from AdvanceSoft Corporation

This study was founding of the project by the Japan Ministry of Economy, Trade and Industry (METI), the New Energy and Industrial Technology Development Organization (NEDO) and Technology Research Association for Future Additive Manufacturing (TRAFAM).

Calculations were performed using the $\mathrm{K}$ computer at RIKEN Advanced Institute for Computational Science, Nagoya University's Fujitsu FX100, and Kyushu University's Fujitsu FX10. The work was supported by the Department of HPC Support,Research Organization for Information Science \& Technology (RIST) under the User, Optimization, and Performance Analysis Support Program for the K computer.

Synchrotron radiation experiments were performed at the BL20XU of SPring-8 with the approval of the Japan Synchrotron Radiation Research Institute (JASRI) (Proposal No. 2015B1840, 2016B1817, and 2017B1594).

\section{References}

Beucher, S., The watershed transformation applied to image segmentation, SCANNING MICROSCOPY-SUPPLEMENT- (1992),pp.299-299.

Fukuyama,H., PROSPECT, Journal of the Society of Instrument and Control Engineers, Vol.54, No.5 (2015), pp. 303-307 (in Japanese).

Guo,W., Cahn, S. B., Nikkel, J. A., Vinen, W. F., and McKinsey, D. N., Visualization study of counterflow in superfluid 
helium-4 using metastable helium molecules, Physical Review Letters, Vol. 105 (2010) 045301.

Khairallah, S. A., Anderson, A. T., Rubenchik, A. M., King. E., Laser powder-bed fusion additive manufacturing: Physics of complex melt flow and formation mechanisms of pores, spatter, and denudation zones, Acta Marerialia, Vol. 108 (2016), pp.36-45.

Kijima,S., Metal alloy physical property value calculation software JMatPRo, SOKEIZAI, Vol. 56, No.12 (2015)pp. 46-51. (in Japanese).

King, W. E., Anderson, A. T., Ferencz, R. M., Hodge, N. E., Kamath C., Khairallah S. A., and Rubenchik A. M., Laser powder bed fusion additive manufacturing of metals; physics, computational, and materials challenges, Applied Physics Reviews, vol.2 (2015), 041304.

Klassen, A., Scharowsky,T. and Körner C.,Evaporation model for beam based additive manufacturing using free surface lattice Boltzmann methods, Journal of Physics D: Applied Physics Vol.47(2014) 275303.

Ly,S., Rubenchik, A. M., Khairallah, S. A., Guss G. and Matthews, M. J., Metal vapor micro-jet controls material redistribution in laser powder bed fusion additive manufacturing, Scientific Reports, 7:4085 (2017).

Sahoo, S., Chou, K., Phase-field simulation of microstructure evolution of Ti-6Al-4V in electron beam additive manufacturing process, Additive Manufacturing, Vol. 9, 14 (2016), pp.14-24.

Saunders, N., Fahrmann, M. and Small, C. J., in Superalloys 2000, ed. Green, K. A., Pollock, T. M. and Kissinger, R. D. p.803, TMS, Warrendale (2000).

Suzuki,Y., Uesugi, K., Takimoto, N., Fukui,T., Aoyama, K., Takeuchi, A., Takano,H., Yagi, N., Mochizuki, T., Goto „S., Takeshita, K., Takahashi, S., Ohashi H., Furukawa,Y., Ohata, T., Matsushita, T., Ishizawa, Y., Yamazaki, H., Yabashi, M., Tanaka, T., Kitamura, H., Ishikawa T., Construction and commissioning of A $248 \mathrm{~m}$ - long beamline with X - ray undulator light source, AIP Conference Proceedings. Vol. 705(2004),p.344.

Takada,S.,Current research on the fluid-dynamics limit based on molecular gas dynamics, Nagare, Vol.19 (2000) pp.226-236 (in Japanese).

Tan, W., Bailey, N. S. and Shin, Y. C., Investigation of keyhole plume and molten pool based on a three-dimensional dynamic model with sharp interface formulation, Journal of Physics D: Applied Physics, Vol. 46, No. 5 (2013) 055501 .

Tomiyama, A., Kataoka, I., Zun, I., Sakaguchi, T., Drag coefficients of single bubbles under normal and micro gravity conditions, JSME International Journal Series B, Fluids and Thermal Engineering, vol. 41 (1998) Issue 2, pp.472-479.

Watanabe, M, Adachi, M., Fukuyama, H., Densities of Fe-Ni melts and thermodynamic correlations, Journal Material Science Vol. 51 (2016), pp. 3303-3310.

Yano, T., On gas flow with evaporation and condensation, Manufacturing \& technology, Vol.60, No.3, (2008) pp.61-65 (in Japanese). 